\title{
Resource predictability and host specificity in fleas: the effect of host body mass
}

\author{
B. R. KRASNOV ${ }^{1 *}$, S. MORAND ${ }^{2}$, D. MOUILLOT ${ }^{3}$, G. I. SHENBROT', I. S. KHOKHLOVA \\ and R. POULIN ${ }^{5}$ \\ ${ }^{1}$ Ramon Science Center and Mitrani Department of Desert Ecology, Facob Blaustein Institutes for Desert Research, \\ Ben-Gurion University of the Negev, P.O. Box 194, Mizpe Ramon 80600, Israel \\ ${ }^{2}$ Center for Biology and Management of Populations, Campus International de Baillarguet, \\ CS 3001634988 Montferrier-sur-Lez cedex, France \\ ${ }^{3}$ UMR CNRS-UMII 5119 Ecosystemes Lagunaires, University of Montpellier II, CC093, \\ FR-34095 Montpellier Cedex 5, France \\ ${ }^{4}$ Desert Animals Adaptations and Husbandry, Wyler Department of Dryland Agriculture, \\ Facob Blaustein Institutes for Desert Research, Ben-Gurion University of the Negev, Beer Sheva 84105, Israel \\ ${ }^{5}$ Department of Zoology, University of Otago, P.O. Box 56, Dunedin, New Zealand
}

(Received 22 November 2005; revised 28 December 2005; accepted 24 Fanuary 2006; first published online 28 March 2006)

\section{SUMMARY}

Ecological specialization is hypothesized to result from the exploitation of predictable resource bases. For parasitic organisms, one prediction is that parasites of large-bodied host species, which tend to be long-lived, should specialize on these hosts, whereas parasites of small host species, which represent more ephemeral and less predictable resources, should become generalists. We tested this prediction by quantifying the association between the level of host specificity of fleas and the mean body mass of their mammalian hosts, using published data from 2 large, distinct geographical regions (South Africa and northern North America). In general, we found supporting evidence that flea host specificity, measured either as the number of host species exploited or their taxonomic distinctness, became more pronounced with increasing host body mass. There were, however, some discrepancies among the results depending on the different measures of host specificity, the geographical region studied, or whether we used the raw values or phylogenetically independent contrasts. These are discussed with respect to other forces acting on the evolution of host specificity in parasites, as well as in the context of the regions' contrasting evolutionary histories. Overall, though, our findings indicate that the exploitation of large-bodied, and therefore long-lived, host species has promoted specialization in fleas, most likely because these hosts represent predictable resources.

Key words: fleas, host body size, host specificity, mammals, North America, South Africa.

\section{INTRODUCTION}

Host specificity is one of the most important characteristics of a parasite species. Although host specificity can vary to some degree among different populations of the same parasite species across its geographical range, it still represents a true character of a parasite species, being less variable within parasite species than among parasite species (Krasnov et al. 2004a). This suggests that the level of host specificity is subjected to natural selection rather than merely reflecting the local restrictions caused by a variety of ecological, morphological, chemical and/ or genetic factors (Fox and Morrow, 1981).

From an evolutionary perspective, selection for higher or lower levels of host specificity (or, in a broader sense, ecological specialization) is affected by a variety of both parasite (=forager)-related and host

* Corresponding author: Ramon Science Center, P.O. Box 194, Mizpe Ramon 80600, Israel. Tel: +972 86588764. Fax: +9728 6586369. E-mail: krasnov@bgu.ac.il
(= resource)-related factors (Fox and Morrow, 1981; Futuyama and Moreno, 1988; Fry, 1996; Desdevises et al. 2002). In particular, the models of Ward (1992) suggested that species tend to specialize on predictable resources, i.e. resources that are relatively stable in both space and time. This likely minimizes extinction rate for an optimal forager.

The main resource for a parasitic species is its host, which provides a parasite with food, habitat and mating grounds. Consequently, specialization in parasites is expected to be associated with the level of predictability of its host resources (Ward, 1992). Persistence of a host individual in time, in turn, is associated with its size. In general, larger host species live longer and, thus, represent a more predictable resource for a parasite (Peters, 1983). In addition, larger hosts offer more niches for parasites. As a result, larger host species are expected to harbour mainly parasite species with higher host specificity, whereas small-bodied hosts should be exploited mainly by generalist parasites. Indeed, it has been shown that larger fish species harbour more species 
of specialist monogenean ectoparasites compared with smaller fish species (Sasal et al. 1999; Simkova et al. 2001).

Here, we address this issue in fleas, which are common ectoparasites of higher vertebrates. The overwhelming majority of fleas parasitize mammals (more than $94 \%$ of species; Vatschenok, 1988), whereas their association with birds is much weaker. Fleas usually alternate between periods when they occur on the body of their hosts and periods when they occur in their hosts' burrows or nests. In most cases, the pre-imaginal development is entirely offhost. The degree of association between a particular flea species and a particular host species varies, with flea species ranging from highly host-specific to host-opportunistic (Marshall, 1981).

We searched for an association between the level of flea specialization and the predictability of the host resource, by relating the level of host specificity of fleas with the mean body mass of their hosts, using published data on host occurrences of fleas from 2 large, distinct geographical regions. Among mammals, a positive correlation between body mass and life-span has been repeatedly demonstrated (Peters, 1983; Brown, 1995). Consequently, we expected that the degree of host specificity of a flea species would be positively correlated with mean body mass of its hosts. We defined host specificity as the number, but also the identity, of host species that are exploited by a parasite species. In other words, rather than just taking the mere number of host species used by a flea as a measure of host specificity, we also applied a measure of host specificity that takes into account the taxonomic or phylogenetic affinities of the various host species (Poulin and Mouillot, 2003). This measure places the emphasis on the taxonomic distance between host species used by a flea rather than on their number, providing a different and complementary perspective on host specificity, one that truly focuses on the specialization of the flea for its host habitat.

\section{MATERIALS AND METHODS}

Data on the host range of fleas were obtained from published regional monographs on fleas from the entire spectrum of mammalian and avian hosts of South Africa (Segerman, 1995) and the northern part of North America (Canada, Alaska and Greenland; Holland, 1985; hereafter referred to as North America). Only mammalian hosts and their fleas were used in the analyses. These regions were selected because (a) both of them are not isolated but instead represent parts of larger contiguous biogeographical regions and (b) their mammalian faunas are mostly composed of different representatives of the same mammalian orders. We cross-checked the species lists with the catalogues of Lewis and Lewis (1990a) and Medvedev et al. (2005) to resolve cases of synonymy. Bats (Chiroptera) represent an exception to the general rule of a positive association between body size and life-span: bats live relatively long despite their small size (Jurgens and Protero, 1987; Wilkinson and South, 2002). In general, bat life-span is about $3 \cdot 5$ times longer than that of other mammals of comparable body sizes (Wilkinson and South, 2002). Consequently, bats and their specific fleas (family Ischnopsyllidae) were excluded from the analysis.

For each flea species, 2 measures of host specificity were used: (1) the number of mammalian species on which the flea species was found, and (2) the specificity index, $S_{\mathrm{TD}}$, and its variance $\operatorname{Var} S_{\mathrm{TD}}$ (Poulin and Mouillot, 2003). The number of hosts examined often covaries with the number of parasite individuals and species found in a survey (Morand and Poulin, 1998). As a result, unequal between-host study effort may result in confounding variation in estimates of flea host specificity. However, the sources used did not provide data on the number of host individuals examined and the number of recorded fleas as is usually the case with regional monographs. Consequently, it was not possible to account for the potential bias related to unequal sampling effort. In addition, parasites characteristic for a prey species can often be transferred to a predator species during predator/prey interaction (Marshall, 1981). As a result, a false parasite-host association can be recorded. To avoid this, we excluded flea species characteristic of rodents and insectivores from the lists of fleas recorded on carnivores based on information on the typical hosts of a flea species from the respective sources. For example, a total of 5 flea species (Echidnophaga gallinacea, Ctenocephalides connatus, Ctenocephalides felis, Synosternus caffer and Xenopsylla erilli) were recorded on the banded mongoose Mungo mungo in South Africa (Segerman, 1995). However, we only considered the former 3 flea species as 'true' parasites of this host because the normal hosts of $S$. caffer and X. erilli are springhare (Pedetes capensis) and the Cape ground squirrel (Xerus inaurus), respectively (Segerman, 1995).

The index $S_{\mathrm{TD}}$ measures the average taxonomic distinctness of all host species used by a parasite species. When these host species are placed within a taxonomic hierarchy, the average taxonomic distinctness is simply the mean number of steps up the hierarchy that must be taken to reach a taxon common to 2 host species, computed across all possible pairs of host species (see Poulin and Mouillot, 2003 for details). For any given host species pair, the number of steps corresponds to half the path length connecting two species in the taxonomic tree, with equal step lengths of one being postulated between each level in the taxonomic hierarchy. The greater the taxonomic distinctness between host species, the higher the number of steps needed, and the higher the value of the index $S_{\mathrm{TD}}$ : thus it is actually 
inversely proportional to specificity. A high index value means that on average the hosts of a flea species are not closely related. Using the taxonomic classification of Wilson and Reeder (1993), all mammal species included here were fitted into a taxonomic structure with 5 hierarchical levels above species, i.e. genus, subfamily, family, order, and class (Mammalia). The maximum value that the index $S_{\text {TD }}$ can take (when all host species belong to different orders) is thus 5 , and its lowest value (when all host species are congeners) is 1 . However, since the index cannot be computed for parasites exploiting a single host species, we assigned a $S_{\mathrm{TD}}$ value of 0 to these flea species, to reflect their strict host specificity. The variance in $S_{\mathrm{TD}}, \operatorname{Var} S_{\mathrm{TD}}$, provides information on any asymmetries in the taxonomic distribution of host species (Poulin and Mouillot, $2003)$; it can only be computed when a parasite exploits 3 or more host species (it always equals zero with 2 host species). To calculate $S_{\mathrm{TD}}$ and $\operatorname{Var} S_{\mathrm{TD}}$, $\mathrm{DM}$ and RP have developed a computer programme using Borland $\mathrm{C}++$ Builder 6.0 (available at http://www.otago.ac.nz/zoology/downloads/poulin/ TaxoBiodiv1.2). All 3 measures (number of host species, $S_{\mathrm{TD}}$ and $\operatorname{Var} S_{\mathrm{TD}}$ ) are, thus, inversely indicative of host specificity. Estimates of $S_{\mathrm{TD}}$ and $\operatorname{Var} S_{\mathrm{TD}}$ were affected by the number of exploited hosts $\left(r^{2}=0 \cdot 1-0 \cdot 43, F=6 \cdot 4-72 \cdot 7\right.$ for South Africa and $r^{2}=0 \cdot 1-0 \cdot 53, F=14 \cdot 8-155 \cdot 2$ for North America; $P<0.002$ for all). Consequently, in subsequent analyses we substituted the original values of $S_{\mathrm{TD}}$ and $\operatorname{Var} S_{\mathrm{TD}}$ by residuals of the regression of logtransformed values of $S_{\mathrm{TD}}$ and $\operatorname{Var}_{\mathrm{TD}}$ against the log-transformed number of exploited hosts.

Our approach was 2-fold. First, for each flea species, we calculated the mean body mass of all exploited host species. Data on mean mammalian body mass were obtained from Silva and Downing (1995). To test for the correlation between flea host specificity and host body mass, we regressed logtransformed measures of host specificity (after controlling for host number for $S_{\mathrm{TD}}$ and $\operatorname{Var} S_{\mathrm{TD}}$ ) against log-transformed values of mean host body mass across flea species, separately for South Africa and North America. This approach determined whether specific fleas do indeed exploit larger host species than generalist fleas; but another way of addressing the same issue is to determine whether large-bodied host species harbour more host-specific fleas than small-bodied ones. Second, therefore, we calculated the mean host specificity (mean number of host species, mean $S_{\mathrm{TD}}$ and mean $\operatorname{Var} S_{\mathrm{TD}}$ ) across all flea species recorded on a host, for each host species. Then, we regressed log-transformed measures of mean flea host specificity (after controlling for host number for $S_{\mathrm{TD}}$ and $\operatorname{Var} S_{\mathrm{TD}}$ ) against logtransformed values of body mass, across all host species, separately for each geographical region. The analyses were carried out using both conventional
Table 1. Summary of regression analyses of the relationship between flea host specificity and mean host body mass in South Africa and North America

\begin{tabular}{lllll}
\hline \hline \multirow{2}{*}{ Region } & $\begin{array}{l}\text { Measure of host } \\
\text { specificity }\end{array}$ & $r^{2}$ & $F$ & $P$ \\
\hline South Africa & Number of hosts & $0 \cdot 01$ & $0 \cdot 93$ & $0 \cdot 33$ \\
& $S_{\mathrm{TD}}$ & $0 \cdot 004$ & $0 \cdot 31$ & $0 \cdot 58$ \\
& $\operatorname{Var}_{\mathrm{TD}}$ & $0 \cdot 09$ & $6 \cdot 5$ & $0 \cdot 01$ \\
North America & Number of hosts & $0 \cdot 001$ & $0 \cdot 1$ & $0 \cdot 7$ \\
& $S_{\mathrm{TD}}$ & $0 \cdot 001$ & $0 \cdot 01$ & $0 \cdot 9$ \\
& $\operatorname{VarS}_{\mathrm{TD}}$ & $0 \cdot 05$ & $5 \cdot 7$ & $0 \cdot 01$ \\
\hline \hline
\end{tabular}

regression and the method of phylogenetically independent contrasts (Felsenstein, 1985).

The method of independent contrasts was used to control for the possible effects of either flea or mammal phylogenies (Felsenstein, 1985; Harvey and Pagel, 1991). The phylogenetic tree for fleas was based on a detailed morphological taxonomy (see details in Krasnov et al. 2004a). The phylogenetic tree for mammals was based on various sources (see details in Morand and Poulin, 1998; Matthee and Robinson, 1999; Krasnov et al. 2004b, c; Flynn et al. 2005). The initial branch length for both trees was set to $1 \cdot 0$. To compute independent contrasts, we used the PDAP:PDTREE module (Garland et al. 1993; Midford et al. 2005) implemented in Mesquite Modular System for Evolutionary Analysis (Maddison and Maddison, 2005). Procedures for the analyses followed Garland et al. (1992, 1993) and Pagel (1992).

Because we examined the correlations of 3 measurements of host specificity with a single independent variable, we avoided an inflated Type I error by performing Bonferroni adjustments of the $\alpha$-level. Significance is only recorded at the adjusted level.

\section{RESULTS}

There were 85 flea species exploiting 105 mammalian host species in South Africa, and 131 fleas exploiting 137 mammalian host species in North America. Six flea species only occurred in both regions (Xenopsylla cheopis, Pulex irritans, Ctenocephalides canis, Ctenocephalides felis, Leptopsylla segnis and Nosopsyllus fasciatus). The body mass of host species ranged from $0.006 \mathrm{~kg}$ (Mus minutoides) to $160 \mathrm{~kg}$ (Panthera leo) in South Africa and from $0.003 \mathrm{~kg}$ (Sorex hoyi) to $200 \mathrm{~kg}$ (Ursus arctos) in North America.

In both regions, flea host specificity in terms of either the number of exploited host species or their taxonomic distinctness $\left(S_{\mathrm{TD}}\right)$ did not correlate with mean body mass of the hosts (Table 1). On the contrary, flea host specificity measured as the taxonomic asymmetry of the host spectrum $\left(\operatorname{VarS}_{\mathrm{TD}}\right)$ decreased significantly with an increase in the mean body mass of exploited hosts (Table 1, Fig. 1). However, no 

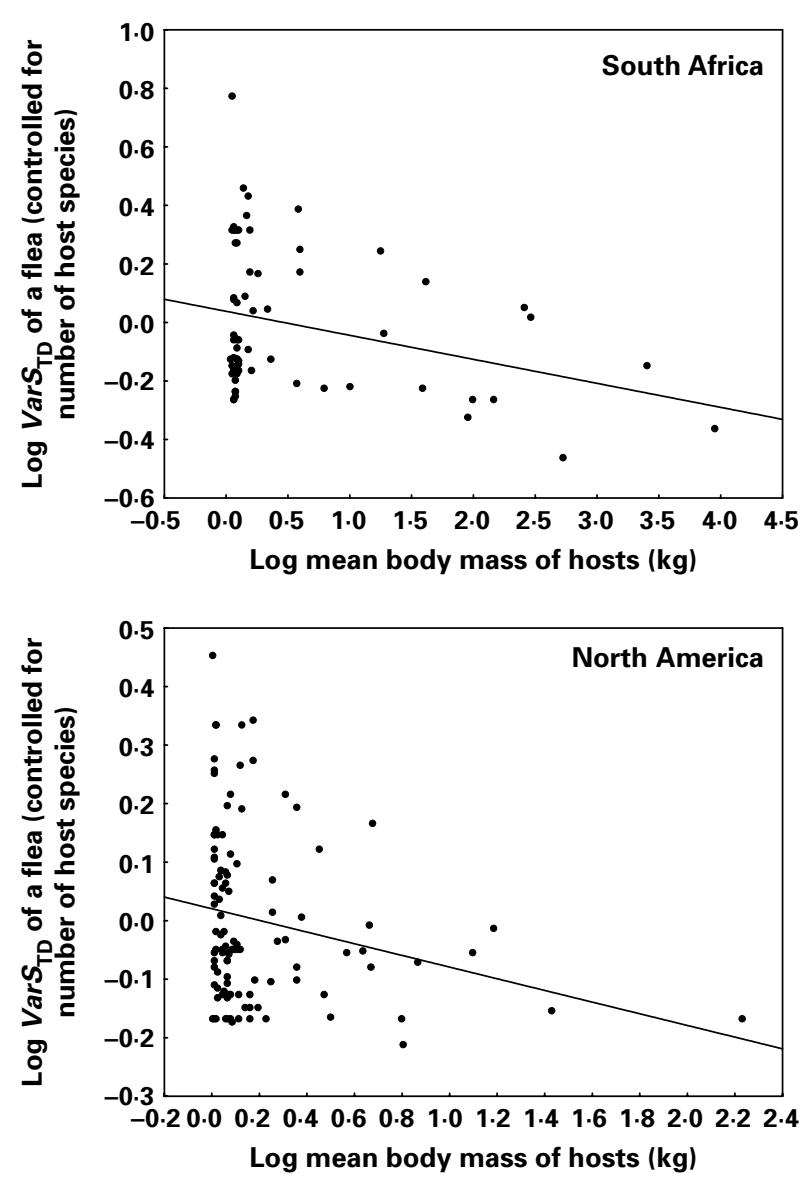

Fig. 1. Relationship between the mean body mass of host species exploited by a flea and the taxonomic asymmetry $\left(\operatorname{Var}_{\mathrm{TD}}\right)$ of these hosts, across flea species from two different geographical regions.

correlation was found between any of the host specificity measures and mean body mass of hosts in either of the two regions when the data were controlled for the confounding effect of phylogeny (Table 2).

When we instead determined whether largebodied host species harbour more host-specific fleas than small-bodied ones, i.e. when we considered the mean number of hosts exploited by the fleas of a host's assemblage, it was negatively correlated with this host's body mass $\left(r^{2}=0 \cdot 20, F=26 \cdot 7\right.$ for South Africa and $r^{2}=0.05, F=6.8$ for North America; $P<0.01$ for both) (Fig. 2). The mean taxonomic diversity of the host spectrum of fleas exploiting a host species correlated positively with body mass of this host in South Africa $\left(r^{2}=0 \cdot 06, F=6 \cdot 2, P<0 \cdot 01\right)$, but no relationship between these two parameters was found for the North American data $\left(r^{2}=0.03\right.$, $F=3 \cdot 4, P=0 \cdot 07$ ) (Fig. 3). In both regions, the body mass of a host was negatively correlated with the mean taxonomic asymmetry of the host spectrum of the fleas that this host harboured $\left(r^{2}=0 \cdot 11, F=13 \cdot 0\right.$ for South Africa and $r^{2}=0 \cdot 17, F=28 \cdot 2$ for North America; $P<0 \cdot 001$ for both; Fig. 4).
Table 2. Summary of the correlation analyses of the relationship between flea host specificity and mean host body mass in South Africa and North America using phylogenetically independent contrasts

\begin{tabular}{llrl}
\hline \hline \multirow{2}{*}{ Region } & $\begin{array}{l}\text { Measure of host } \\
\text { specificity }\end{array}$ & \multicolumn{1}{l}{$r$} & $P$ \\
\hline South Africa & Number of hosts & $0 \cdot 01$ & $0 \cdot 9$ \\
& $S_{\mathrm{TD}}$ & $0 \cdot 06$ & $0 \cdot 6$ \\
& VarS $_{\mathrm{TD}}$ & $-0 \cdot 09$ & $0 \cdot 4$ \\
North America & Number of hosts & $0 \cdot 17$ & $0 \cdot 06$ \\
& $S_{\mathrm{TD}}$ & $0 \cdot 08$ & $0 \cdot 3$ \\
& Var $_{\mathrm{TD}}$ & $-0 \cdot 07$ & $0 \cdot 4$ \\
\hline \hline
\end{tabular}
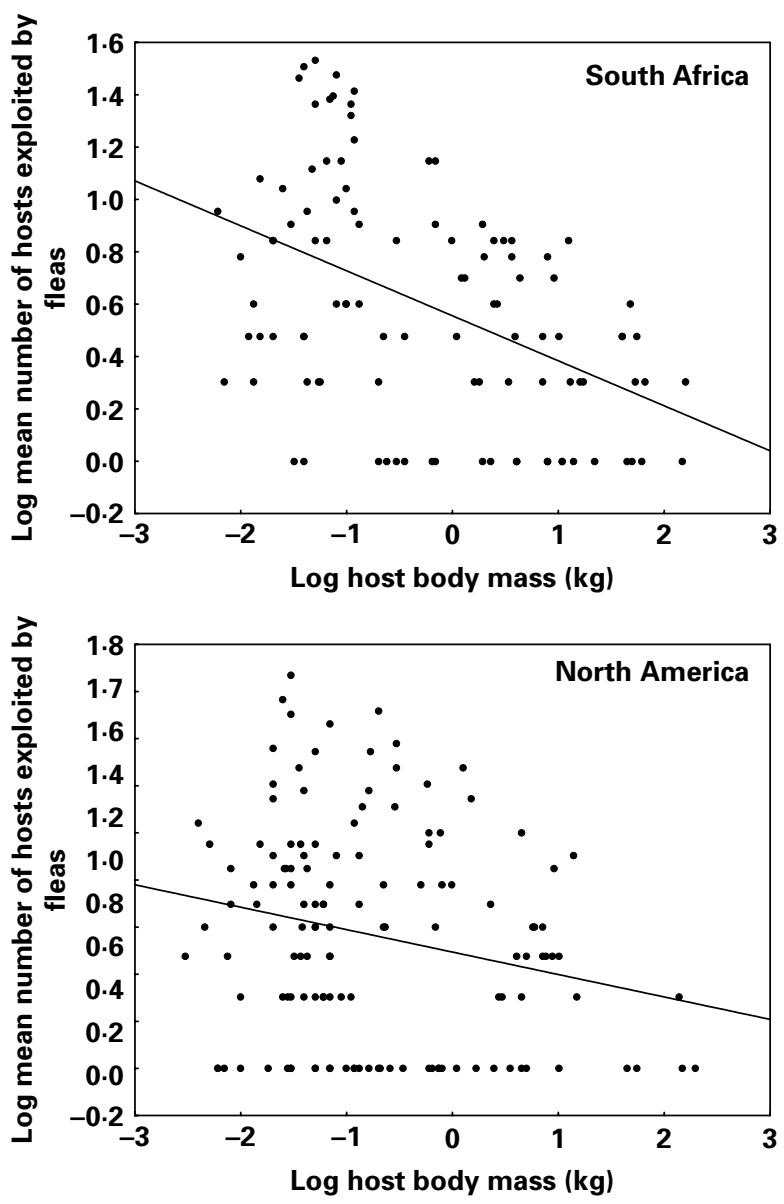

Fig. 2. Relationship between body mass of a host and mean number of hosts exploited by the fleas harboured by this host, across host species from two different geographical regions.

Relationships between host body mass and measurements of host specificity of fleas harboured by a host were only partly supported by the method of independent contrasts. In South African hosts, contrasts in host body mass were not correlated with contrasts in either mean number or mean $S_{\mathrm{TD}}$ of hosts exploited by the fleas composing an assemblage $(r=-0 \cdot 11$ and $r=0 \cdot 007$, respectively, $P>0 \cdot 2$ for 

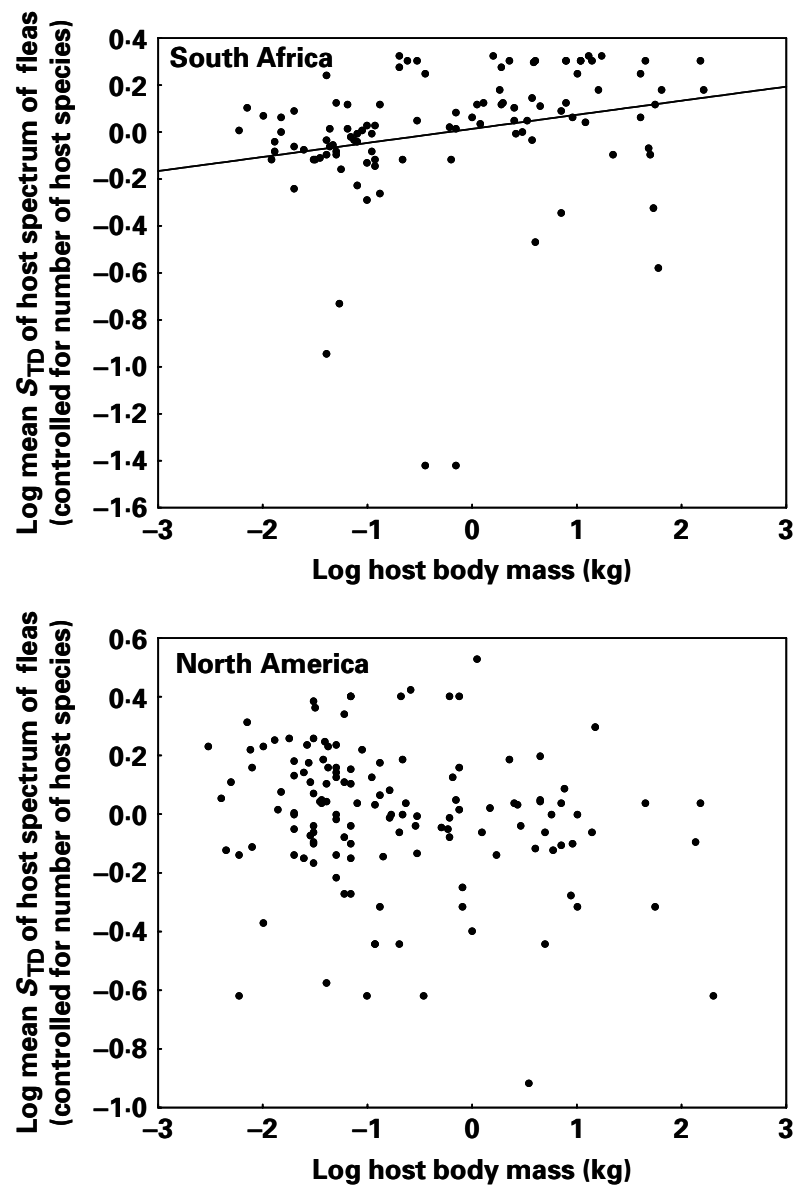

Fig. 3. Relationship between body mass of a host and the mean taxonomic diversity $\left(S_{\mathrm{TD}}\right)$ of the host spectrum exploited by the fleas harboured by this host, across host species from two different geographical regions.

both). However, contrasts in host body mass correlated negatively with contrasts in mean $\operatorname{Var}_{\mathrm{TD}}$ of hosts exploited by a host's fleas $(r=-0 \cdot 24, P<0 \cdot 01)$. In North America, contrasts in host body mass were negatively correlated with contrasts in the mean number of hosts used by a host's fleas $(r=-0 \cdot 21$, $P<0 \cdot 01)$, but no relationship was found between host body mass and either mean $S_{\mathrm{TD}}$ or mean $\operatorname{Var} S_{\mathrm{TD}}$ of hosts exploited by a host's fleas $(r=0 \cdot 12$ and $r=-0 \cdot 01$, respectively, $P>0 \cdot 1$ for both).

\section{DISCUSSION}

The results of this study demonstrate a weak but consistent association between the level of flea host specificity and host body mass. However, this association was not always supported by the method of independent contrasts and was somewhat differently expressed in the two geographical regions.

The association between flea host specificity and host body mass was revealed mainly when host specificity was considered in terms of the number of exploited host species and their taxonomic asymmetry. The latter parameter describes how much taxonomic heterogeneity there is among a group of
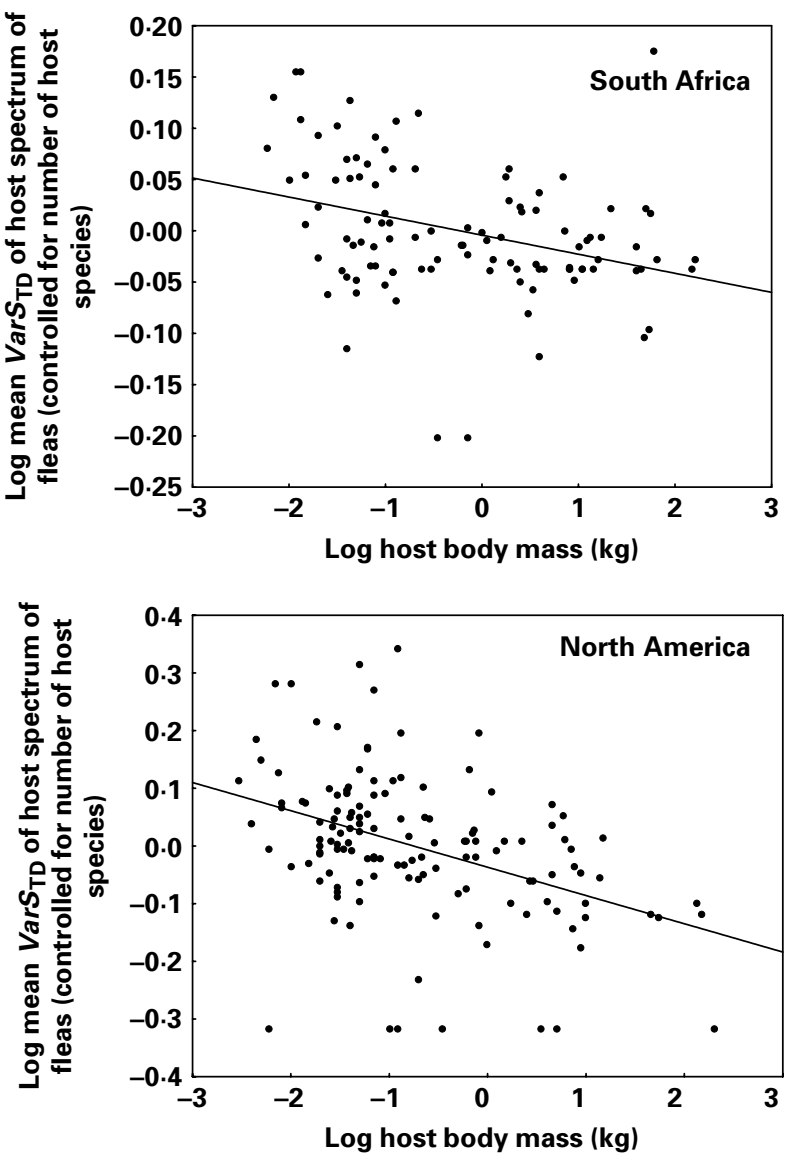

Fig. 4. Relationship between body mass of a host and the mean taxonomic asymmetry $\left(\operatorname{Var}_{\mathrm{TD}}\right)$ of the host spectrum exploited by the fleas harboured by this host, across host species from two different geographical regions.

host species (Warwick and Clarke, 2001 ; Poulin and Mouillot, 2003). A high variance usually means that one main branch in the taxonomic tree of host species contributes proportionally more species to the list than other branches. For example, Oropsylla labis and Corypsylla kohlsi exploit 4 hosts each. However, all hosts of $O$. labis belong to the same subfamily (Sciurinae) and 3 of them are congenerics (Spermophilus columbianus, Spermophilus franklinii and Spermophilus richardsonii). On the contrary, hosts of $C$. kohlsi belong to 2 different orders (Insectivora and Rodentia). They are represented by 3 congeneric insectivores (Sorex obscurus, Sorex pacificus and Sorex trowbridgii) and 1 rodent (Microtus townsendii). As a result, $\operatorname{Var}_{\mathrm{TD}}$ values for these species differ sharply $(0 \cdot 25$ versus 4$)$. The relationships between host body mass and flea host specificity showed a similar pattern when considered from both the host and flea perspectives. In general, small-bodied hosts harboured mainly hostopportunistic fleas (with a high number and/or low taxonomic 'evenness' of exploited hosts), whereas host-specific fleas tend to use larger hosts. This finding supports the observations of Sasal et al. (1999), Simkova et al. (2001) and Desdevises et al. 
(2002) for fish-monogenean systems and suggests some role for the predictability of resources in the evolution of flea host specificity. Indeed, large hosts live longer than smaller hosts and, thus, represent more permanent sources of food and living space for fleas. As a result, specialization can be favoured in fleas exploiting larger and, thus, more predictable hosts. This supports the hypothesis about the link between specialization and predictability of the resource (Ward, 1992). However, host predictability as a resource for fleas can be manifested in other ways than host body size. Indeed, some small colonial hosts (ground squirrels, prairie dogs, whistling rats, meerkats) can represent highly predictable resources because their colonies may persist in a location for a long time, i.e. longer than the life-span of an individual host. In addition, the relatively high frequency of body-to-body contact in small colonial hosts increases the predictability of the resource in terms of transmission probability compared with many large hosts which are solitary. Nevertheless, the majority of small mammals are solitary rather than colonial. Consequently, the higher predictability of small colonial hosts as a resource for fleas could possibly weaken the relationship between host body size and flea host specificity found in this study, but it is unlikely to reverse the direction of this relationship.

Another measure of the resource predictability, host abundance, has been suggested to influence the evolution of host specificity in parasites (Norton and Carpenter, 1998). However, this appeared not to be the case for fleas and mammalian hosts because larger mammals are usually less abundant (i.e. lower population density) than smaller mammals (Peters, 1983). The explanation can be that the effect of relative host abundance on parasite specificity should depend on parasite dispersal abilities (Reed and Hafner, 1997; Desdevises et al. 2002). If these abilities are relatively high, then the relative effect of host abundance on specificity should likely be low. Indeed, fleas can transfer from host to host by free movement or during between-host contacts. Individual fleas can disperse rather long distances (Bates, 1962; Marshall, 1981; Kuznetzov et al. 1999). Direct contact between different host species has also been shown to ensure host-to-host flea transfer (Krasnov and Khokhlova, 2001). On the other hand, the probability of host-to-host transfer decreases with a decrease in host density. Therefore, the probability of colonization of a new host is likely lower for a flea that parasitizes larger hosts than for a flea that parasitizes smaller hosts. The relatively lower degree of host specificity in fleas exploiting large mammals in comparison with fleas exploiting small mammals can be a result of this difference in colonization probability.

Larger hosts may include a greater variety of niches (Kuris et al. 1980). Different fleas prefer different host body areas when they occur on relatively large hosts (Muller et al. 2001 ; Hsu et al. 2002). For example, Echidnophaga myrmecobii preferred to settle on the head and body of the rabbit Oryctolagus cuniculus, whereas Echidnophaga perilis occurred mainly on the fore- and hind-feet (Shepherd and Edmonds, 1979). Although different parts of a host body provide the same basic resource for fleas (i.e. host blood), the pattern of acquisition of this resource by fleas can differ among these body parts. Skin thickness, capillary depth and defensibility differ among body parts of a mammal, and these differences are likely more pronounced in larger than in smaller species. As a result, flea specialization can be facilitated in larger hosts (Kuris et al. 1980). The negative correlation between host body mass and mean number of hosts exploited by a host's fleas supports this explanation.

The negative but triangular distribution of points in the relationship between body mass and flea host specificity implies that smaller hosts can harbour both host-specific and host-opportunistic fleas, whereas larger hosts harbour mainly host-specific fleas. For example, the mean number of hosts exploited by 6 fleas recorded on Malacotrix typica (body mass about $100 \mathrm{~g}$ ) is $25 \cdot 2$ and by 8 fleas recorded on Parotomys littledalei (body mass about $130 \mathrm{~g}$ ) is $11 \cdot 3$, whereas that of 2 fleas recorded on Potamochoerus porcus (body mass about $54 \mathrm{~kg}$ ) is 11.5. This, as well as the relative weakness of the association between host body size and flea host specificity, suggests that resource predictability alone cannot explain the patterns and pathways of the evolution of host specificity in fleas. Other factors must be involved as well. For example, a trade-off between the ability to use many host species and the average fitness achieved in these hosts is expected (Poulin, 1998). This is because close adaptation to one host species may be likely achieved at the expense of adaptations to other host species due to the presumably high cost of parasite adaptations against multiple host defence mechanisms. However, this appeared not to be the case for fleas, as host opportunistic flea species were shown to be also the ones that attain higher local abundances on their hosts (Krasnov et al. 2004c). On the contrary, fleas using either many host species or taxonomically diverse host species achieve not only greater average abundance, but also a broader geographical range than the more host-specific fleas (Krasnov et al. 2005). The reason for this can be that the advantages of having many host species and therefore a broader geographical range prevails over the physiological costs of adaptations against the hosts' antiparasitic defences. Another factor affecting the evolution of host specificity in fleas is related to the variation in the offhost environment (Krasnov et al. 2004a). In general, environmental conditions, measured as departures from average conditions across the geographical 
range of a flea, influence all measures of flea host specificity even if the host specificity of flea species has been shown to be a true species character moreor-less repeatable across populations of a flea species (Krasnov et al. 2004a). The effect of environmental variables on the degree of local host specificity of a flea is likely related to the microclimatic preferences of the pre-imaginal stages. For example, the flea Xenopsylla ramesis parasitizes several host species (mainly gerbils) throughout the Middle East (Lewis and Lewis, 1990b). However, in some areas Meriones crassus is dropped out of the host spectrum of this flea (Krasnov et al. 1997). The reason for this is the unsuitability of microclimatic and substrate conditions in $M$. crassus burrows for the successful survival of eggs, larvae and newly-emerged imago of $X$.ramesis (Krasnov et al. 2001).

Furthermore, the local availability of taxonomically-related hosts can affect, to some extent, the level of host specificity of a given flea species. Among parasites of fish, for instance, parasites exploiting hosts belonging to species-rich taxa have been found to be less host specific than parasites exploiting hosts belonging to species-poor taxa (Poulin, 1992; Barker et al. 1994; Sasal et al. 1998).

Finally, the different pattern in the specificity-host body size relationship between South Africa and North America calls for some explanation. Indeed, in most cases when the relationship was found, it was stronger for South African than for North American flea-mammal associations. This difference may be associated with the differences between these two regions during the Cenozoic when the main evolutionary development of flea-mammal associations occurred (Medvedev, 2005). Glaciations with repeated advances and retreat of ice-sheets were characteristic for North America and not for South Africa, especially during the Quarternary. Glaciation-interglaciation cycles may have lead to the repetitive break-ups and restorations of associations between flea and host species in North America, whereas these associations in South Africa were likely more stable on a geological time-scale. The 'historical instability' of the relationships between flea and host species in North America could have resulted in a relatively weak association between flea and host traits.

In conclusion, the results of this study demonstrated that predictability of resources measured as host body size matters to the evolution of parasite host specificity. A stable and predictable host resource will select for greater specialization in parasites. However, the predictability of host resources alone cannot explain all patterns of host specificity among flea species.

We thank two anonymous referees for their helpful comments on an earlier version of the manuscript. This study was partly supported by the Israel Science
Foundation (Grant no. $249 / 04$ to B.R.K.). This is publication no. 199 of the Ramon Science Center and no. 509 of the Mitrani Department of Desert Ecology.

\section{REFERENCES}

Barker, S. C., Cribb, T. H., Bray, R. A. and Adlard, R. D. (1994). Host-parasite associations on a coral reef: pomacentrid fishes and digenean trematodes.

International Fournal for Parasitology 24, 643-647.

Bates, J. K. (1962). Field studies on the behaviour of bird fleas. I. Behaviour of the adults of three species of bird fleas in the field. Parasitology 52, 113-132.

Brown, J. H. (1995). Macroecology. University of Chicago Press, Chicago.

Desdevises, Y., Morand, S. and Legendre, P. (2002). Evolution and determinants of host specificity in the genus Lamellodiscus (Monogenea). Biological Fournal of the Linnean Society 77, 431-443.

Felsenstein, J. (1985). Phylogenies and the comparative method. American Naturalist 125, 1-15.

Flynn, J., Finarelli, J. A., Zehr, S., Hsu, J. \& Nedball, M. A. (2005). Molecular phylogeny of the Carnivora (Mammalia): assessing the impact of increased sampling on resolving enigmatic relationships. Systematic Biology 54, 317-337.

Fox, L. R. and Morrow, P. A. (1981). Specialization: species property or local phenomenon? Science 211, 887-893.

Fry, J. D. (1996). The evolution of host specialization: are trade-offs overrated? American Naturalist 148, S84-S107.

Futuyma, D. J. and Moreno, G. (1988). The evolution of ecological specialization. Annual Review of Ecology and Systematics 19, 207-233.

Garland, T. Jr., Harvey, P. H. and Ives, A. R. (1992). Procedures for the analysis of comparative data using phylogenetically independent contrasts. American Naturalist 41, 18-32.

Garland, T. Jr., Dickerman, A. W. C., Janis, M. and Jones, J. A. (1993). Phylogenetic analysis of covariance by computer simulation. Systematic Biology 42, 265-292.

Harvey, P. H. and Pagel, M. D. (1991). The Comparative Method in Evolutionary Biology. Oxford University Press, Oxford.

Holland, G. P. (1985). The fleas of Canada, Alaska and Greenland (Siphonaptera). Memoirs of the Entomological Society of Canada 130, 1-631.

Hsu, M.-H., Hsu, T.-C. and Wu, W.-J. (2002). Distribution of cat fleas (Siphonaptera: Pulicidae) on the cat. Fournal of Medical Entomology 39, 685-688.

Jurgens, K. D. and Prothero, J. (1987). Scaling of maximal lifespan in bats. Comparative Biochemistry and Physiology A 88, 361-367.

Krasnov, B. R. and Khokhlova, I. S. (2001). The effect of behavioural interactions on the exchange of flea (Siphonaptera) between two rodent species. Fournal of Vector Ecology 26, 181-190.

Krasnov, B. R., Shenbrot, G. I., Medvedev, S. G., Vatschenok, V. S. and Khokhlova, I. S. (1997). Hosthabitat relation as an important determinant of spatial distribution of flea assemblages (Siphonaptera) on rodents in the Negev Desert. Parasitology 114, 159-173. 
Krasnov, B. R., Khokhlova, I. S., Fielden, L. J. and Burdelova, N. V. (2001). The effect of temperature and humidity on the survival of pre-imaginal stages of two flea species (Siphonaptera: Pulicidae). Fournal of Medical Entomology 38, 629-637.

Krasnov, B. R., Mouillot, D., Shenbrot, G. I., Khokhlova, I. S. and Poulin, R. (2004a). Geographical variation in host specificity of fleas (Siphonaptera): the influence of phylogeny and local environmental conditions. Ecography 27, 787-797.

Krasnov, B. R., Shenbrot, G. I., Khokhlova, I. S. and Degen, A. A. (2004b). Flea species richness and parameters of host body, host geography and host “milieu” Fournal of Animal Ecology 73, 1121-1128.

Krasnov, B. R., Poulin, R., Shenbrot, G. I., Mouillot, D. and Khokhlova, I. S. $(2004 c)$. Ectoparasitic “jacks-of-all-trades": relationship between abundance and host specificity in fleas (Siphonaptera) parasitic on small mammals. American Naturalist 164, 506-516.

Krasnov, B. R., Poulin, R., Shenbrot, G. I., Mouillot, D. and Khokhlova, I. S. (2005). Host specificity and geographic range in haematophagous ectoparasites. Oikos 108, 449-456.

Kuris, A. M., Blaustein, A. R. and Alio, J. J. (1980). Hosts as islands. American Naturalist 116, 570-586.

Kuznetzov, A. A., Matrosov, A. N., Chyong, L. T. V. and Dat, D. T. (1999). Movements of the synantropous rats and their fleas in the settlements of the southern Vietnam. Problemy Osobo Opasnyh Infektzyi [Problems of Especially Dangerous Diseases] 79, 59-65 (in Russian).

Lewis, R. E. and Lewis, H. H. (1990a). Catalogue of Invalid Genus-Group and Species-Group Names in Siphonaptera (Insecta), Theses Zoologocae, v. 11. Koeltz Scientific Books, Koenigstein.

Lewis, R. E. and Lewis, J. H. (1990b). An annotated checklist of the fleas (Siphonaptera) of the Middle East. Fauna of Saudi Arabia 11, 251-277.

Maddison, W. P. and Maddison, D. R. (2005). Mesquite: a Modular System for Evolutionary Analysis. Version 1.06. http://mesquiteproject.org.

Marshall, A. G. (1981). The Ecology of Ectoparasite Insects. Academic Press., London.

Matthee, C. A. and Robinson, T. J. (1999). Cytochrome $b$ phylogeny of the family bovidae: resolution within the alcelaphini, antilopini, neotragini, and tragelaphini. Molecular Phylogenetics and Evolution 12, 31-46.

Medvedev, S. G. (2005). An Attempt of a System Analysis of the Evolution of the Order of Fleas (Siphonaptera), Lectures in Memoriam N. A. Kholodkovsky, No. 57. Russian Entomological Society and Zoological Institute of Russian Academy of Sciences, Saint-Petersburg (in Russian).

Medvedev, S. G., Lobanov, A. L. and Lyanguzov, I. A. (2005). World database of fleas (Nov 2004 version). In: Species 2000 and ITIS Catalogue of Life : 2005 Annual Checklist (ed. Bisby, F. A., Ruggiero, M. A., Wilson, K. L., Cachuela-Palacio, M., Kimani, S. W., Roskov, Y. R., Soulier-Perkins, A. and van Hertum, J.). Species 2000, Reading (CD-ROM).

Midford, P. E., Garland, T. Jr. and Maddison, W. (2005). PDAP:PDTREE Package for Mesquite, version 1.07. http://mesquiteproject.org/pdap_mesquite/index. html
Morand, S. and Poulin, R. (1998). Density, body mass and parasite species richness of terrestrial mammals. Evolutionary Ecology 12, 717-727.

Muller, G. H., Kirk, R. W., Scott, D. W., Miller, W. H., Griffin, C. E. and Miller, W. T. (2001). Muller and Kirk's Small Animal Dermatology. Saunders, Philadelphia.

Norton, D. A. and Carpenter, M. A. (1998). Mistletoe as parasites: host specificity and speciation. Trends in Ecology and Evolution 13, 101-105.

Pagel, M. D. (1992). A method for the analysis of comparative data. Fournal of Theoretical Biology 156, 431-442.

Peters, R. H. (1983). The Ecological Implications of Body Size. Cambridge University Press, Cambridge.

Poulin, R. (1992). Determinants of host specificity in parasites of freshwater fishes. International Fournal for Parasitology 22, 753-758.

Poulin, R. (1998). Large-scale patterns of host use by parasites of freshwater fishes. Ecology Letters 1, 118-128.

Poulin, R. and Mouillot, D. (2003). Parasite specialization from a phylogenetic perspective: a new index of host specificity. Parasitology 126, 473-480.

Reed, D. L. \& Hafner, M. S. (1997). Host specificity of chewing lice on pocket gophers: a potential mechanism for cospeciation. Fournal of Mammalogy 78, 655-660.

Sasal, P., Desdevises, Y. and Morand, S. (1998). Hostspecialization and species diversity in fish parasites: phylogenetic conservatism? Ecography 21, 639-645.

Sasal, P., Trouvé, S., Muller-Graf, C. and Morand, S. (1999). Specificity and host predictability: a comparative analysis among monogenean parasites of fish. Fournal of Animal Ecology 68, 437-444.

Segerman, J. (1995). Siphonaptera of Southern Africa: handbook for the identification of fleas. Publications of the South African Institute for Medical Research 57, $1-264$.

Shepherd, R. C. H. and Edmonds, J. W. (1979). The distribution of the stickfast fleas, Echidnophaga mirmecobii Rotschild and E. perilis Jordan, on the wild rabbit, Oryctolagus cuniculus (L.). Australian Fournal of Zoology 27, 261-271.

Silva, M. and Downing, J. A. (1995). CRC Handbook of Mammalian Body Masses. CRC Press, Boca Raton, Florida.

Simkova, A., Desdevises, Y., Gelnar, M. and Morand, S. (2001). Morphometric correlates of host specificity in Dactylogyrus species (Monogenea) parasites of European Cyprinid fish. Parasitology 123, 169-177.

Vatschenok, V. S. (1988). Fleas - Vectors of Pathogens Causing Diseases in Humans and Animals. Nauka Publishing House, Leningrad, USSR (in Russian).

Ward, S. A. (1992). Assessing functional explanations of host-specificity. American Naturalist 139, 883-891.

Warwick, R. M. and Clarke, K. R. (2001). Practical measures of marine biodiversity based on relatedness of species. Oceanography and Marine Biology Annual Review 39, 207-231.

Wilkinson, G. S. and South, J. M. (2002). Life history, ecology and longevity of bats. Aging Cell 1, 124-131.

Wilson, D. E. and Reeder, D. M. (eds.) (1993). Mammal Species of the World: A Taxonomic and Geographic Reference. Smithsonian Institution Press, Washington. 